\section{\$22. Kinetic Effects of Inclined Magnetic Field on Physical Sputtering by Impurity lons}

Kawamura, G., Tomita, Y., Kirschner, A. (Forschungszentrum Jülich, Germany)

Impurity ions coming from SOL to divertor are primary source of sputtered impurities in transport simulation code including $\mathrm{ERO}^{1)}$. That implies the importance of sputtering yield by the background impurities in plasma. However, their flux and incident angle distribution have to be calculated from fluid quantities like density and temperature. An enhancement effect of inclined magnetic field on sputtering yield by hydrogen bombardment was clarified in our previous work ${ }^{2}$. We developed a particle tracing code to obtain incident angle and energy distribution in pre-calculated electric field by PIC simulation to investigate the effect on impurity ions. The size of the simulation box is chosen to be much larger than the Larmor radius of impurity ions and length of magnetic presheath region. Collision and anomalous transport are not taken into account because the simulation box is much shorter than the mean free path. The code calculates the flux distribution on the surface as a function of impact energy and angle and calculates the sputtering yield from a sputtering model ${ }^{3)}$.

In order to solve the motion of impurity ions, electric field at each position in a sheath region is necessary to calculate force acting on them. Inclined magnetic field leads to polarization drift caused by variation of electric field due to parallel motion of the particle and hence a transitional region with electric field, i.e. magnetic presheath, is formed. The length of the presheath is several times larger than the ion Larmor radius and causes significant change on the velocity distribution of ions. We assume that the impurity density is much lower than that of deuterium ions and thus the impurity does not disturb the plasma. We employ a 1D PIC (ParticleIn-Cell) code to simulate a deuterium plasma.

We carried out PIC simulation to obtain the electric field in the magnetic presheath and Debye sheath, and then investigated the incident angle and sputtering yield by the impurity tracing code. Since we use constant background electric field, the calculation is much faster than the PIC code and thus we can obtain clearer results in much less time. The plasma parameters used here are taken from measurements in TEXTOR device ${ }^{1)}$ as follows; electron temperature, $T_{\mathrm{e}}=40 \mathrm{eV}$, normalized ion Larmor radius, $\rho_{\mathrm{i}} / \lambda_{\mathrm{De}}=20$, magnetic field angle, $\varphi=0^{\circ}, 75.5^{\circ}, 88.2^{\circ}$, ion-electron temperature ratio of the plasma source in the SOL, $T_{\mathrm{i}} / T_{\mathrm{e}}=1$, and magnetic flux density, $B=2.25 \mathrm{~T}$.

We calculate the sputtering yield from the incident velocity of the ions. We simulated the following combinations; deuterium $[+1]$, carbon $[+1]$ and carbon $[+4]$ ions on a carbon wall in Fig. 1(a), carbon[+1], tungsten[+1] and tungsten[+4] ions on a tungsten wall in Fig. 1(b). The self sputtering causes
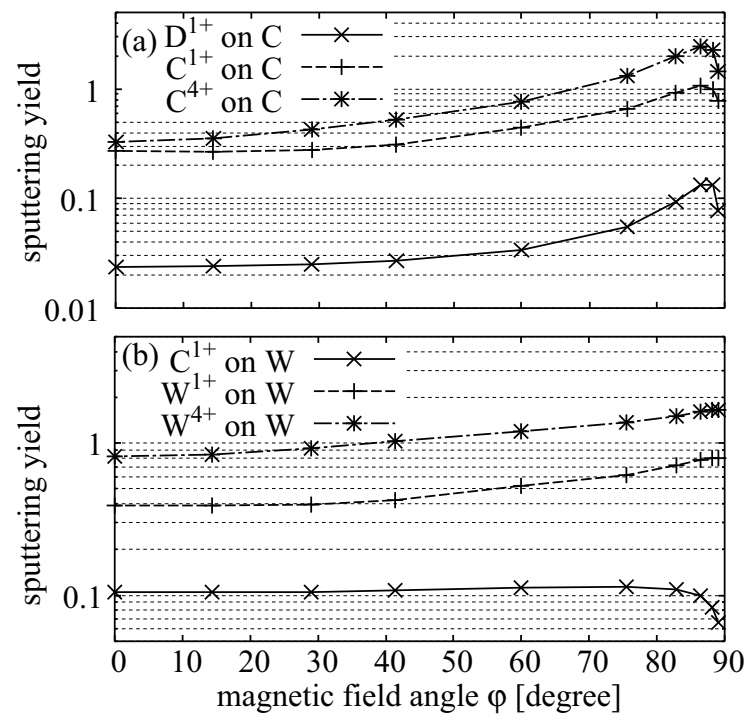

Fig. 1: Dependence of sputtering yield on magnetic field angle $\varphi$; (a) deuterium [+1], carbon[+1] and carbon[+4] ions on carbon, (b) carbon[+1], tungsten[+1] and tungsten[+4] ions on tungsten.

much larger sputtering yield than the other two cases, D on C and $\mathrm{D}$ on $\mathrm{W}$. The enhancement due to the magnetic field is observed for $\varphi>30^{\circ}$ in the two cases of self sputtering. The increase of carbon sputtering by deuterium is observed for $\varphi>50^{\circ}$. Tungsten sputtering by carbon does not have the enhancement but significant decrease of the yield for $\varphi>85^{\circ}$. On the other hand, the tungsten self sputtering does not have the decrease near $\varphi=90^{\circ}$ because of smaller incident angle than the other lighter projectiles. Comparisons of the charge state are also shown in Fig. 1. The profile is not sensitive to the charge state but the yield becomes large because of the high energy gain due to the large electrostatic force.

We conclude from above and other analyses that the parameters with most prominent effect on the incident angle are the magnetic field angle and the mass of the projectile. Simple formulation of these effect is difficult and therefore simulations are necessary to obtain the effective yield used in impurity transport simulation codes. Simulations, however, involve uncertainties in models such as background electric field and initial velocity distribution of ions. Evaluation of influence of these models and parameters used in the model must be made before using results in an impurity transport simulation. Application of the sputtering yield model presented here to an impurity transport simulation code, ERO, and validation of results with experiments are future issues.

1) A. Kirschner et al., Nucl. Fusion 40 (2000) 989.

2) G. Kawamura et al., J. Nucl. Mater., 415 (2011) S192

3) W. Eckstein, Sputtering Yields, Springer Berlin 2007, pp. $33-186$ 